\title{
Structural Basis for Reduced Glomerular Filtration Capacity in Nephrotic Humans
}

\author{
M. Claudia Drumond, * Batya Kristal, ${ }^{\ddagger}$ Bryan D. Myers, ${ }^{\star}$ and William M. Deen \\ ${ }^{*}$ Department of Chemical Engineering, Massachusetts Institute of Technology, Cambridge, Massachusetts $02139 ;$ and ${ }^{\ddagger}$ Nephrology \\ Division, Stanford University School of Medicine, Stanford, California 94305
}

\section{Abstract}

Previous studies have established that in a variety of human glomerulopathies the reduced glomerular filtration rate (GFR) is due to a marked lowering of the ultrafiltration coefficient $\left(K_{f}\right)$. To identify the factors which lower $K_{f}$, we measured the filtering surface area per glomerulus, filtration slit frequency, basement membrane thickness, and GFR and its determinants in patients with minimal change and membranous nephropathies and in age-matched healthy controls. Overall values of $K_{f}$ for the two kidneys were calculated from GFR, renal plasma flow rate, systemic colloid osmotic pressure, and three assumed values for the transcapillary pressure difference. "Experimental" values of the glomerular hydraulic permeability $\left(k_{\text {exp }}\right)$ were then calculated from $K_{f}$, glomerular filtering surface area, and estimates of the total number of nephrons of the two kidneys. Independent estimates of the glomerular hydraulic permeability ( $\left.k_{\text {model }}\right)$ were obtained using a recent mathematical model that is based on analyses of viscous flow through the various structural components of the glomerular capillary wall. Individual values of basement membrane thickness and filtration slit frequency were used as inputs in this model. The results indicate that the reductions of $K_{f}$ in both nephropathies can be attributed entirely to reduced glomerular hydraulic permeability. The mean values of $\boldsymbol{k}_{\exp }$ and $\boldsymbol{k}_{\text {model }}$ were very similar in both disorders and much smaller in the nephrotic groups than in healthy controls. There was good agreement between $\boldsymbol{k}_{\text {exp }}$ and $\boldsymbol{k}_{\text {model }}$ for any given group of subjects. It was shown that, in both groups of nephrotics, filtration slit frequency was a more important determinant of the water flow resistance than was basement membrane thickness. The decrease in filtration slit frequency observed in both disorders caused the average path length for the filtrate to increase, thereby explaining the decreased hydraulic permeability. (J. Clin. Invest. 1994. 94:1187-1195.) Key words: membranous nephropathy • minimal change nephropathy - ultrafiltration coeficient • hydraulic permeability • glomerular capillary morphometry

Address correspondence to William M. Deen, Department of Chemical Engineering, 66-509, Massachusetts Institute of Technology, Cambridge, MA 02139 .

Received for publication 6 December 1993 and in revised form 31 May 1994.

J. Clin. Invest.

(C) The American Society for Clinical Investigation, Inc.

0021-9738/94/09/1187/09 \$2.00

Volume 94, September 1994, 1187-1195

\section{Introduction}

Studies of a variety of human glomerulopathies have demonstrated that the overall ultrafiltration coefficient $\left(K_{\mathrm{f}}\right)^{1}$ for the two kidneys is reduced relative to that in healthy controls (1$5)$. Although the inability to measure the glomerular transcapillary hydraulic pressure difference $(\Delta P)$ in humans precludes precise calculations of $K_{\mathrm{f}}$, the reductions in $K_{\mathrm{f}}$ computed for nephrotic individuals are typically so large that there is little doubt that glomerular ultrafiltration capacity is severely compromised in such disorders. Because $K_{\mathrm{f}}$ as determined in these human studies is the product of the hydraulic permeability of the glomerular capillary wall $(k)$ and the total capillary surface area available for filtration in the two kidneys, the observed reductions in $K_{\mathrm{f}}$ might be due to decreased $k$, decreased surface area per glomerulus, a decreased number of functioning glomeruli, or some combination of these factors. Measurements have been reported for some of the key quantities in humans which should influence $K_{\mathrm{f}}$, including surface area per glomerulus (1, $2,4,6)$, basement membrane thickness $(1-4,6,7)$, and filtration slit frequency, which is the number of filtration slits per unit length of peripheral capillary wall (1-4). Reductions in filtration slit frequency reflect broadening and "effacement" of the epithelial foot processes, a uniform finding in virtually all humans with the nephrotic syndrome, regardless of its etiology. The most consistent inference from morphometric analysis of glomeruli of nephrotic individuals has been a strong correlation between decreases in estimated $K_{\mathrm{f}}$ and reductions in filtration slit frequency $(8-11)$.

Efforts to ascertain whether changes in filtration slit frequency or other factors can account for the observed alterations in $K_{\mathrm{f}}$ have been hampered by an inadequate understanding of the relationship between the various structural quantities and $k$. These relationships have been clarified recently by a mathematical model of the glomerular capillary wall, which is based on detailed analyses of viscous flow across the various structural components $(12,13)$. The structures include the endothelial fenestrae, glomerular basement membrane, and filtration slits. Using as inputs certain morphometric data from rats and the hydraulic permeability measured for isolated rat glomerular basement membrane, this "ultrastructural model" was able to predict a value for $k$ that is in good agreement with micropuncture measurements in normal rats (13). It was also able to explain changes in $k$ in an experimental rat model of glomerular injury, suggesting that it might be useful in interpreting data in human glomerular diseases.

1. Abbreviations used in this paper: $k$, hydraulic permeability of the glomerular capillary wall; $K_{\mathrm{f}}$, ultrafiltration coefficient; MAP, mean arterial pressure; PAH, para-aminohippuric acid; RPF, rate of plasma flow. 
The objective of this study was to apply the ultrastructural model to membranous nephropathy and minimal change nephropathy, two human glomerulopathies in which $K_{\mathrm{f}}$ is estimated to decline markedly, despite preservation of the surface area available for filtration $(1,10)$. To elucidate the biophysical basis for reduced GFR and $K_{\mathrm{f}}$ in these disorders, we combined an assessment of glomerular filtration dynamics with a morphometric analysis of glomeruli obtained by renal biopsy.

\section{Methods}

\section{Patient population}

The subjects of our study were 34 consecutive adult patients below 60 $\mathrm{yr}$ of age, who were referred to one of us (B. D. Myers) because of a nephrotic syndrome and a histopathological diagnosis of either membranous nephropathy $(n=23)$ or minimal change nephropathy $(n=11)$. They were between 18 and $59 \mathrm{yr}$ old, and 20 of the 34 were male. 36 healthy volunteers, matched for age (21-54) and gender (19 males), served as controls. They were divided into two groups. Healthy control group $1(n=24)$ underwent a physiological evaluation of glomerular function. Healthy control group $2(n=12)$ were living kidney transplant donors, who underwent a morphometric analysis of glomeruli obtained by kidney biopsy at the time of transplantation. Each control subject denied a history of renal disease, hypertension, or diabetes and was found to be normotensive and normoglycemic and to have a negative dipstick test for urinary protein at the time of evaluation.

\section{Physiologic evaluation}

Patients and healthy control group 1 consented to undergo differential solute clearances according to a protocol which had been approved previously by the Institutional Review Board at the Stanford University School of Medicine. Each was admitted to a Clinical Research Center on the morning of the study. Antihypertensive agents were withdrawn $48 \mathrm{~h}$ before admission in all patients receiving such therapy. Urine was voided spontaneously after diuresis had been established with an oral water load $(10-15 \mathrm{mg} / \mathrm{kg})$. A priming dose of inulin $(50 \mathrm{mg} / \mathrm{kg}$ ) and para-aminohippuric acid (PAH, $12 \mathrm{mg} / \mathrm{kg}$ ) was then administered. Thereafter, inulin and PAH were given by continuous infusion to maintain plasma levels at 20 and $1.5 \mathrm{mg} / \mathrm{dl}$, respectively.

$60 \mathrm{~min}$ after the priming dose, arterial blood pressure was determined, and blood was sampled for examination of systemic colloid osmotic pressure $\left(\pi_{\mathrm{A}}\right)$ and plasma concentrations of albumin and IgG. Four timed urine collections were then made, each of which was bracketed by a blood sample drawn from a peripheral vein. GFR was expressed as the average value for the four timed inulin clearances. The rate of plasma flow (RPF) was estimated by dividing the corresponding clearance of PAH by an estimate of the prevailing renal arteriovenous extraction ratio for PAH. We have shown previously that reductions of GFR and peritubular capillary protein concentration exert an additive effect to lower the extraction ratio for PAH in patients with glomerular disease (14). Based on the observed relationships, we assigned a value for the extraction ratio of PAH of 0.9 to healthy controls and 0.8 or 0.7 to the nephrotic patients with a normalized GFR above or below $80 \mathrm{ml} /$ min per $1.73 \mathrm{~m}^{2}$, respectively.

The concentrations of inulin and PAH were determined with an automated assay (10). The concentrations of endogenous albumin and IgG in serum and urine were determined immunochemically, and the colloid osmotic pressure of plasma was determined by membrane osmometry, as described previously (15).

The GFR, RPF, and $\pi_{\mathrm{A}}$ were used together with the model of Deen et al. (16) to calculate values of $K_{\mathrm{f}}$ for each individual, expressed as the total $K_{\mathrm{f}}$ for all nephrons in the two kidneys. Because the glomerular transcapillary hydraulic pressure difference $(\Delta P)$ could not be measured, we used assumed values of 35,40 , and $45 \mathrm{mmHg}$; the lower values are representative of micropuncture results in normal rats (17), whereas the highest value corresponds to moderate glomerular hypertension. The fractional clearances of albumin $\left(\theta_{\mathrm{alb}}\right)$ and $\operatorname{IgG}\left(\theta_{\mathrm{lgG}}\right)$ were determined by dividing the clearances of albumin and IgG, respectively, by that of inulin. The fractional clearances of albumin and IgG were measured in only 21 of the 24 individuals of healthy control group 1 .

\section{Morphometric measurements}

Light microscopy. The biopsies of all nephrotic patients were performed contemporaneously with clearance determinations and before the initiation of specific treatment. Paraffin-embedded tissue was sectioned at 1$\mu \mathrm{m}$ intervals and stained with periodic acid-Schiff reagent. On average, 19 glomeruli per biopsy were analyzed at the light microscopic level in each nephrotic patient (range 7-30). The average number of glomeruli among the 12 control biopsies was also 19 (range 13-30). A dedicated computer system (Southern Micro Instruments, Inc., Atlanta, GA), consisting of a video camera, screen, microscope, and digitizing tablet, was used to perform measurements $(1,2)$. The outline of each glomerular tuft in the cross-section was traced onto the digitizing tablet at a magnification of 900, and the cross-sectional area of the tuft $\left(A_{\mathrm{G}}\right)$ was computed using area perimeter analysis. Glomerular volume $\left(V_{G}\right)$ was calculated from the measured $A_{\mathrm{G}}$ and corrected to account for tissue shrinkage associated with paraffin embedding, using a linear shrinkage factor $\left(f_{\mathrm{s}}\right)$ (18):

$V_{\mathrm{G}}=\frac{\beta}{d} A_{\mathrm{G}}{ }^{3 / 2} f_{\mathrm{s}}^{-3}$

where $\beta$ is a dimensionless "shape coefficient" ( $\beta=1.38$ for spheres $)$ and $d$ is a "size distribution coefficient" which is introduced to account for variations in glomerular size (18). We used $d=1.1$ as in previous studies (1-4), which corresponds to a distribution of glomerular sizes with a standard deviation of $\sim 25 \%$ of the mean size (18). We determined that, in our experimental procedure for tissue fixation, the value of the shrinkage factor is $f_{\mathrm{s}}=0.86$, and used this value in Eq. 1 .

The numbers of patent $\left(n_{\mathrm{P}}\right)$ and globally sclerosed $\left(n_{\mathrm{S}}\right)$ glomeruli were counted in sections of cortical tissue. The percentage of sclerosed glomeruli $(G)$ was calculated by

$$
G=\frac{n_{\mathrm{S}}}{n_{\mathrm{S}}+n_{\mathrm{P}} \frac{D_{\mathrm{S}}}{D_{\mathrm{P}}}} \cdot 100
$$

where $D_{\mathrm{P}}$ and $D_{\mathrm{S}}$ are the diameters of patent and sclerosed glomeruli, respectively, which are proportional to the square root of the tuft crosssectional area. The ratio $D_{\mathrm{p}} / D_{\mathrm{S}}$ accounts for the differences in size between patent and sclerosed glomeruli and the consequent difference in the probability of encountering a glomerulus of either type in a random cross-section.

Electron microscopy. For transmission electron microscopy, the tissue was fixed in $2.5 \%$ glutaraldehyde and embedded in epon. Toluidine blue-stained sections were then surveyed to locate the two patent glomeruli closest to the center of each section. Ultrathin sections $(60-70$ $\mathrm{nm}$ ) of the selected glomeruli were next stained with uranyl acetate and lead citrate and photographed. A complete montage of each glomerulus was prepared, and point and intercept counting at a magnification of 2,820 was used to determine the peripheral capillary surface area $(S)$, which was defined as the interface between the peripheral capillary wall and epithelium, and calculated as

$S=s_{\mathrm{V}} V_{\mathrm{G}}$

where $s_{\mathrm{v}}$ is the surface density of peripheral capillary wall (expressed as length of peripheral capillary wall per unit cross-sectional area of glomerulus). Six to eight high-power electron photomicrographs $(\times 11,280)$ were then obtained from each of the two glomerular profiles to evaluate the thickness of the glomerular basement membrane and the frequency of epithelial filtration slits.

The harmonic mean basement membrane thickness $\left(\delta_{\mathrm{bm}}\right)$ was calcu- 


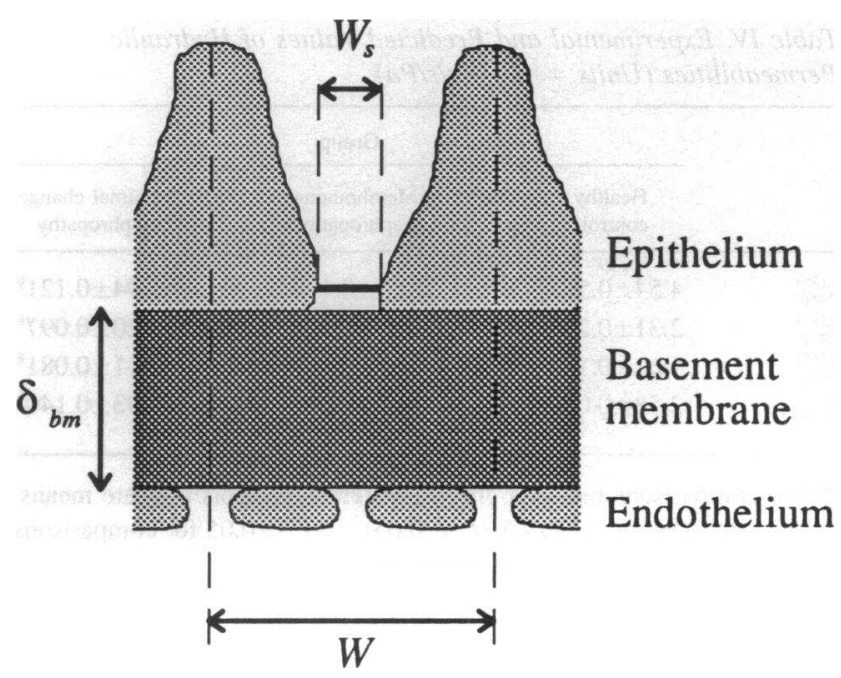

Figure 1. Schematic representation of the glomerular capillary wall showing the fenestrated endothelium, the basement membrane (of thickness $\delta_{\mathrm{bm}}$ ), and the epithelial foot processes connected by the slit diaphragms. The width of a repeating structural unit is $W$, and the width of the slit channel at the level of the slit diaphragm is $W_{s}$.

lated for each individual from the measured (apparent) harmonic mean thickness $\left(\delta_{\mathrm{bm}}^{\prime}\right)$ by

$\delta_{\mathrm{bm}}=\frac{8}{3 \pi} \delta_{\mathrm{bm}}^{\prime}$

where $8 /(3 \pi)$ is a correction factor derived by Jensen et al. (19) to account for the random angle of sectioning. The filtration slit frequency was determined by counting the total number of slits and dividing it by the total length of peripheral capillary wall captured on the electron micrographs $(1,10)$.

\section{Calculation of $k$ from hemodynamic and morphometric data}

$k$ is given by the ultrafiltration coefficient divided by the peripheral capillary surface area. The experimental estimate of $k$ from the hemodynamic data $\left(k_{\text {exp }}\right)$ was obtained from $K_{\mathrm{f}}$, the surface area per glomerulus $(S)$, the percentage of sclerosed glomeruli $(G)$, and the total number of glomeruli in the two kidneys $(n)$ by

$k_{\exp }=\frac{K_{\mathrm{f}}}{n S(1-G / 100)}$.

We assumed $n=2 \times 10^{6}(20)$ for all subjects.

The value of $k_{\text {exp }}$ was calculated for each individual with membranous nephropathy or minimal change nephropathy. To obtain $k_{\text {exp }}$ for healthy controls, we used the individual morphometric data from healthy control group 2 together with the mean hemodynamic data from healthy control group 1.

\section{Calculation of $k$ using ultrastructural model}

Estimates of $k$ independent of those given by Eq. 5 were obtained from filtration slit frequency and basement membrane thickness by using the model of Drumond and Deen (13), in which the capillary wall is approximated as consisting of a large number of repeating structural units. As illustrated in Fig. 1, each structural unit is based on a single filtration slit. The width of a structural unit $(W)$ was calculated from the filtration slit frequency $(F S F)$ by

$W=\frac{2}{\pi} \frac{1}{F S F}$
Table I. Inputs for Ultrastructural Model

\begin{tabular}{lc}
\hline \multicolumn{1}{c}{ Quantity } & Value \\
\hline Permeability of endothelium $\left(k_{\mathrm{en}}\right)$ & $2.0 \times 10^{-7} \mathrm{~m} / \mathrm{s} / \mathrm{Pa}$ \\
Permeability of epithelial slits $\left(k_{\mathrm{s}}\right)$ & $7.9 \times 10^{-8} \mathrm{~m} / \mathrm{s} / \mathrm{Pa}$ \\
Width of structural unit $(W)$ & Individual data \\
Width of epithelial slits $\left(W_{\mathrm{s}}\right)$ & $39 \mathrm{~nm}$ \\
Basement membrane thickness $\left(\delta_{\mathrm{bm}}\right)$ & Individual data \\
Darcy permeability of basement membrane $\left(K_{\mathrm{D}}\right)$ & $2.7 \mathrm{~nm}^{2}$ \\
Fractional area of fenestrae $\left(\epsilon_{\mathrm{f}}\right)$ & 0.2 \\
Number density of fenestrae $\left(\mathrm{n}_{\mathrm{f}} / W\right)$ & $8.33 \times 10^{6} \mathrm{~m}^{-1}$ \\
& \\
\hline
\end{tabular}

where $2 / \pi$ is a correction factor, derived in the Appendix, which accounts for the random angle of sectioning. Using the concept of resistances in series, the overall hydraulic permeability of the capillary wall was calculated from the permeabilities of each layer by

$k_{\text {model }}=\left(\frac{1}{k_{\text {en }}}+\frac{1}{k_{\mathrm{bm}}}+\frac{1}{k_{\mathrm{ep}}}\right)^{-1}$

where $k_{\mathrm{en}}, k_{\mathrm{bm}}$, and $k_{\mathrm{ep}}$ are, respectively, the hydraulic permeabilities of the endothelium, basement membrane, and epithelium. Many of the structural parameters needed appear not to have been measured for the human glomerular capillary wall, so that we used certain values derived from data for rats, as described in detail by Drumond and Deen (12, 13). The resulting permeabilities of the endothelium $\left(k_{\mathrm{en}}\right)$ and of the filtration slits $\left(k_{\mathrm{s}}\right)$ were, respectively, $2.0 \times 10^{-7}$ and $7.9 \times 10^{-8} \mathrm{~m} / \mathrm{s} /$ $\mathrm{Pa}(12,13)$. The permeability of the epithelial layer was calculated using

$k_{\text {ep }}=\epsilon_{\mathrm{s}} k_{\mathrm{s}}=\frac{W_{\mathrm{s}}}{W} k_{\mathrm{s}}$

where $\epsilon_{\mathrm{s}}$ is the fraction of the basement membrane area occupied by filtration slits, and $W_{s}$ is the slit width; $\epsilon_{s}=W_{s} / W$. To be consistent with the use of rat data to compute $k_{\mathrm{s}}$, we used $W_{\mathrm{s}}=39 \mathrm{~nm}$ as for the rat $(12,13,21)$. This value is not very different from an estimate obtained using the data of Ellis et al. (9) for healthy humans $\left(W_{\mathrm{s}} \cong\right.$ $43 \mathrm{~nm})$.

The permeability of the basement membrane $\left(k_{\mathrm{bm}}\right)$ was calculated using Eq. 21 of Drumond and Deen (13). In addition to $\epsilon_{s}$, this equation requires knowledge of the basement membrane thickness $\left(\delta_{\mathrm{bm}}\right)$, the Darcy permeability $\left(K_{\mathrm{D}}\right)$ of the basement membrane, the fraction of the basement membrane area occupied by endothelial fenestrae $\left(\epsilon_{\mathrm{f}}\right)$, and the number of fenestrae per structural unit $\left(n_{\mathrm{f}}\right)$. Whereas $\delta_{\mathrm{bm}}$ was measured in this study, $K_{\mathrm{D}}, \epsilon_{\mathrm{f}}$, and $n_{\mathrm{f}}$ were estimated using values for the rat glomerular capillary wall (13). The inputs for the calculations of $k$ with the ultrastructural model are listed in Table I.

\section{Statistical analysis}

Tabulated results are given as mean \pm standard error. Paired Student $t$ tests and an ANOVA between groups were used to assess the significance of differences in mean values. In all cases, differences between means were judged significant when $P<0.05$. Linear regression analysis was used to test the strength of the relationship between $k_{\text {model }}$ and $k_{\text {exp }}$.

\section{Results}

The results of the hemodynamic measurements and calculations are shown in Table II. There were no statistically significant differences between the two nephrotic disorders in any of the hemodynamic quantities. In both nephrotic groups the values of 
Table II. Mean Values of Functional Results

\begin{tabular}{|c|c|c|c|}
\hline \multirow[b]{2}{*}{ Quantity (units) } & \multicolumn{3}{|c|}{ Group } \\
\hline & Healthy controls & $\begin{array}{l}\text { Membranous } \\
\text { nephropathy }\end{array}$ & $\begin{array}{c}\text { Minimal } \\
\text { change } \\
\text { nephropathy }\end{array}$ \\
\hline GFR (ml/min) & $113 \pm 3 *$ & $75 \pm 8^{\ddagger}$ & $88 \pm 12^{\ddagger}$ \\
\hline $\mathrm{RPF}(\mathrm{ml} / \min )$ & $618 \pm 22 *$ & $736 \pm 85^{*}$ & $635 \pm 70^{*}$ \\
\hline Filtration fraction & $0.185 \pm 0.005^{*}$ & $0.117 \pm 0.009^{\ddagger}$ & $0.140 \pm 0.018^{\prime}$ \\
\hline MAP $(m m H g)$ & $88 \pm 2 *$ & $108 \pm 3^{\ddagger}$ & $102 \pm 4^{\ddagger}$ \\
\hline$\pi_{\mathrm{A}}(\mathrm{mmHg})$ & $23.2 \pm 0.4^{*}$ & $15.0 \pm 0.9^{\ddagger}$ & $12.9 \pm 1.3^{\ddagger}$ \\
\hline$K_{f}^{(35)}(\mathrm{ml} / \mathrm{min} / \mathrm{mmHg})$ & $18.4 \pm 1.6 *$ & $4.7 \pm 0.7^{\ddagger}$ & $5.1 \pm 1.0^{\ddagger}$ \\
\hline $\mathrm{K}_{\mathrm{f}}^{(40)}(\mathrm{ml} / \mathrm{min} / \mathrm{mmHg})$ & $9.3 \pm 0.4^{*}$ & $3.5 \pm 0.4^{\ddagger}$ & $3.9 \pm 0.6^{\ddagger}$ \\
\hline$K_{f}^{(4)}(\mathrm{ml} / \mathrm{min} / \mathrm{mmHg})$ & $6.5 \pm 0.2 *$ & $2.8 \pm 0.3^{\ddagger}$ & $3.1 \pm 0.5^{\ddagger}$ \\
\hline$\theta_{\text {alb }}$ & $(2.8 \pm 1.0) \times 10^{-6 *}$ & $0.010 \pm 0.003^{\ddagger}$ & $0.017 \pm 0.009$ \\
\hline$\theta_{\mathrm{Ig}}$ & $(1.2 \pm 0.1) \times 10^{-6 *}$ & $0.004 \pm 0.001^{\ddagger}$ & $0.011 \pm 0.008$ \\
\hline
\end{tabular}

${ }^{*}$ Different symbols indicate means that are different by ANOVA ( $P$ $<0.05)$.

GFR, filtration fraction, and $\pi_{\mathrm{A}}$ were all significantly depressed relative to those in healthy controls. Conversely, mean arterial pressure (MAP) was elevated in the nephrotic groups. Differences in RPF between nephrotics and controls were not statistically significant. As a consequence of the low GFR and the reduced colloid osmotic pressure opposing filtration, $K_{f}$ was calculated to be much lower in either nephrotic group than in controls. If $\Delta \mathrm{P}$ is assumed to have remained constant at 35$40 \mathrm{mmHg}$, then the reductions in $K_{\mathrm{f}}$ were approximately twoto fourfold. If the systemic hypertension in the nephrotic groups is assumed to have caused a $5-\mathrm{mmHg}$ increase in $\Delta \mathrm{P}$ (from 35 to 40 or from 40 to 45 ), then there is as much as a fivefold reduction in the calculated value of $K_{\mathrm{f}}$. The virtually identical values of $K_{\mathrm{f}}$ in membranous nephropathy and minimal change nephropathy are of particular interest, given the marked differences in morphometry between these groups (see below). Also shown in Table II are fractional clearances of albumin and IgG, which were three to four orders of magnitude higher in the two nephrotic groups than in healthy controls.

Results for the morphometric quantities are shown in Table III. The percentage of globally sclerosed glomeruli was low in

Table III. Mean Values of Morphometric Results

\begin{tabular}{cccc}
\hline & \multicolumn{3}{c}{ Group } \\
\cline { 2 - 4 } & $\begin{array}{c}\text { Healthy } \\
\text { controls }\end{array}$ & $\begin{array}{c}\text { Membranous } \\
\text { nephropathy }\end{array}$ & $\begin{array}{c}\text { Minimal } \\
\text { change } \\
\text { nephropathy }\end{array}$ \\
\hline $\begin{array}{c}\text { Sclerosed glomeruli (\%) } \\
\text { Glomerular volume } \\
\left(10^{6} \mu \mathrm{m}^{3}\right)\end{array}$ & $2.3 \pm 1.2^{*}$ & $6.2 \pm 1.8^{*}$ & $0.73 \pm 0.73^{*}$ \\
$\begin{array}{c}\text { Surface area per } \\
\text { glomerulus }\left(10^{5} \mu \mathrm{m}^{2}\right)\end{array}$ & $2.97 \pm 0.32^{*}$ & $5.37 \pm 0.46^{\ddagger}$ & $4.06 \pm 0.61^{* \ddagger}$ \\
$\begin{array}{c}\text { Filtration slit frequency } \\
(V m m)\end{array}$ \\
$\begin{array}{c}\text { Basement membrane } \\
\text { thickness }(n m)\end{array}$ & $1370 \pm 49^{*}$ & $384 \pm 46^{\ddagger}$ & $315 \pm 60^{\ddagger}$ \\
\hline
\end{tabular}

${ }^{*} \pm$ Different symbols indicate means that are different by ANOVA $(P$ $<0.05)$.
Table IV. Experimental and Predicted Values of Hydraulic Permeabilities (Units $=10^{-9} \mathrm{~m} / \mathrm{s} / \mathrm{Pa}$ )

\begin{tabular}{llll}
\hline & \multicolumn{3}{c}{ Group } \\
\cline { 2 - 4 } & $\begin{array}{l}\text { Healthy } \\
\text { controls }\end{array}$ & $\begin{array}{c}\text { Membranous } \\
\text { nephropathy }\end{array}$ & $\begin{array}{c}\text { Minimal change } \\
\text { nephropathy }\end{array}$ \\
\hline$k_{\text {exp }}^{(35)}$ & $4.54 \pm 0.52^{* \ddagger}$ & $0.748 \pm 0.139^{\S}$ & $0.794 \pm 0.121^{\S}$ \\
$k_{\text {exp }}^{(40)}$ & $2.31 \pm 0.27^{*}$ & $0.561 \pm 0.100^{\S}$ & $0.620 \pm 0.097^{\S}$ \\
$k_{\text {exp }}^{(45)}$ & $1.61 \pm 0.19^{*}$ & $0.451 \pm 0.079^{\ddagger 8}$ & $0.511 \pm 0.081^{\S}$ \\
$k_{\text {model }}$ & $2.58 \pm 0.08^{*}$ & $0.733 \pm 0.081^{8}$ & $0.693 \pm 0.140^{\S}$ \\
\hline
\end{tabular}

${ }^{*} 8$ For comparisons between groups, different symbols indicate means that are different by ANOVA $(P<0.05)$. ${ }^{\ddagger} P<0.05$ for comparisons of $k_{\text {exp }}$ with $k_{\text {model }}$ within a given group.

all three groups, and the numerical differences in the mean values between groups were not significant. Due to a significant increase in glomerular volume in membranous nephropathy, the value for the surface area per glomerulus in this disorder was markedly enhanced. The numerical differences between the mean value of surface area in minimal change nephropathy and that in healthy controls and membranous nephropathy were not significant, while the differences in the mean values of glomerular volume were marginal ( $P=0.044$ for minimal change nephropathy versus healthy controls and $P=0.015$ for minimal change nephropathy versus membranous nephropathy). In membranous nephropathy, there was an approximately threefold reduction in the filtration slit frequency relative to controls, accompanied by a doubling of the basement membrane thickness. The reduction in filtration slit frequency measured in minimal change nephropathy was very similar to that seen in membranous nephropathy, but in minimal change nephropathy the value of the basement membrane thickness remained normal. Thus, the morphometric characteristics of membranous nephropathy differed from those in minimal change nephropathy in that glomerular volume, filtration surface area, and basement membrane thickness were all markedly increased in the former.

The "experimental" estimates of hydraulic permeability ( $k_{\text {exp }}$, Eq. 5) and the predicted values from the ultrastructural model ( $k_{\text {model }}$, Eq. 7) are compared in Table IV. The mean values of $k_{\exp }$ were very similar for membranous nephropathy and minimal change nephropathy at each of the assumed pressures and were much smaller in the nephrotic groups than in healthy controls. There was good agreement between $k_{\text {exp }}$ and $k_{\text {model }}$ for the two nephrotic groups. The differences between $k_{\text {exp }}$ and $k_{\text {model }}$ were statistically significant only for healthy controls when $k_{\exp }$ was evaluated at $\Delta \mathrm{P}=35$ or $45 \mathrm{mmHg}$, and for membranous nephropathy when $k_{\exp }$ was evaluated at $\Delta \mathrm{P}=45$ mmHg.

Individual values of $k_{\text {exp }}$ (evaluated at $\Delta \mathrm{P}=40 \mathrm{mmHg}$, $\left.k_{\text {exp }}^{(40)}\right)$ and $k_{\text {model }}$ for minimal change nephropathy and membranous nephropathy are shown in Fig. 2. There was a significant positive correlation between $k_{\text {exp }}$ and $k_{\text {model }}$ in both groups. The correlation coefficients $(r)$ between $k_{\text {model }}$ and $k_{\text {exp }}^{(35)}$, between $k_{\text {model }}$ and $k_{\text {exp }}^{(40)}$, and between $k_{\text {model }}$ and $k_{\text {exp }}^{(45)}$ were, respectively, $0.45,0.42$, and 0.40 for membranous nephropathy; $0.72,0.65$, and 0.62 for minimal change nephropathy; and $0.49,0.46$, and 0.44 for the combined data of membranous nephropathy and minimal change nephropathy. Meaningful correlation coeffi- 


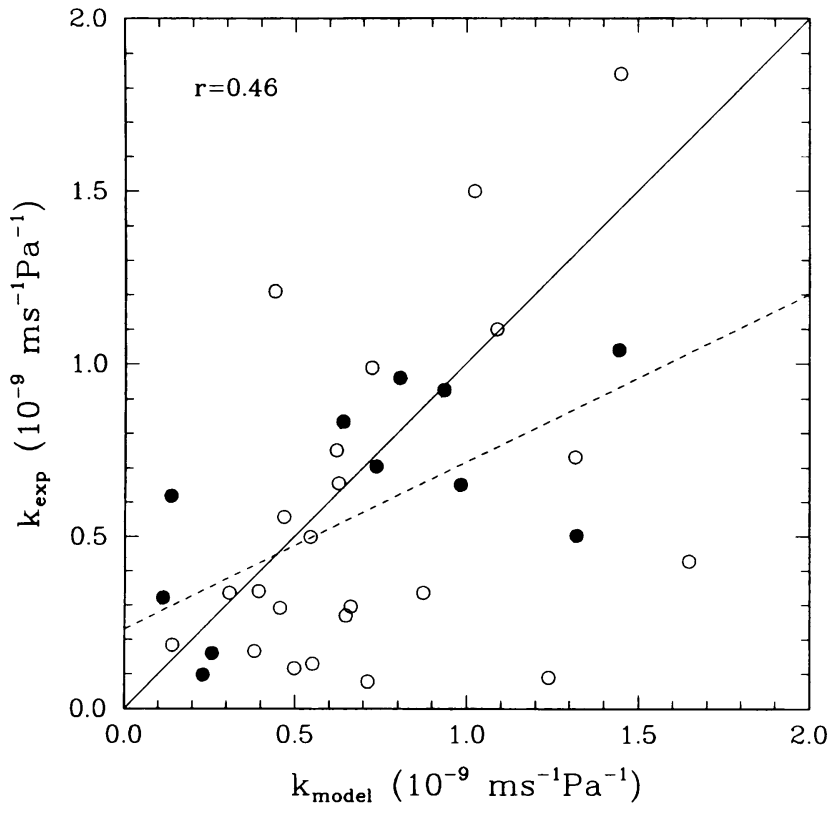

Figure 2. Individual values of $k_{\text {model }}$ and $k_{\text {exp }}$ in membranous (open symbols) and minimal change (filled symbols) nephropathies. For each individual, $k_{\text {exp }}$ was computed at an assumed transcapillary hydraulic pressure difference of $\mathbf{4 0} \mathbf{m m H g}$. The solid line is the identity line, and the dashed line is the regression line for the two groups. The $r$ value for the two groups is also shown.

cients for healthy controls could not be calculated because the functional and morphometric data were obtained in separate groups. Much of the scatter of the points shown in Fig. 2 is likely due to the assumptions regarding $\Delta \mathrm{P}$ and $n$, which significantly affect $k_{\text {exp }}$. Specifically, differences in $\Delta \mathrm{P}$ between individuals within a given group were not taken into account, and $n$ was assumed to be the same for all individuals in all groups. Another potential source of scatter in Fig. 2 is the method by which $S$ was calculated in the present study. Using the Cavalieri method as a gold standard, Lane et al. (22) have reported that determination of $V_{\mathrm{G}}$ by the Weibel method may be unreliable when fewer than 15 glomeruli are available in the biopsy sample. To examine this possible source of error, we calculated $k_{\text {exp }}$ separately in the individuals whose biopsies contained $\geq 15$ glomeruli ( 9 healthy controls, 16 with membranous nephropathy, and 7 with minimal change nephropathy). In these subsets $k_{\text {exp }}^{(40)}$ was similar to the values given in Table IV for the entire groups: $2.58 \pm 0.30$ vs $2.31 \pm 0.27$ for healthy controls; $0.64 \pm 0.13$ vs $0.56 \pm 0.10$ for membranous nephropathy; $0.64 \pm 0.12$ vs $0.62 \pm 0.10$ in minimal change nephropathy (all $10^{-9} \mathrm{~m} / \mathrm{s} / \mathrm{Pa}$ ). None of these differences were statistically significant. Thus, whereas errors in calculated $S$ from biopsies with $<15$ glomeruli could have contributed to the differences between $k_{\text {model }}$ and $k_{\text {exp }}$, such a contribution is likely to have been small. Finally, the assumptions regarding the uniformity of some of the parameters used in the calculation of $k_{\text {model }}$ are also likely to have contributed to the differences between this quantity and $k_{\text {exp }}$. Thus, many factors are likely to account for the scatter in Fig. 2, and better quantitative agreement between individual values of $k_{\text {model }}$ and $k_{\exp }$ would have been difficult to achieve.

Values of $k_{\exp }^{(40)}$ and $k_{\text {model }}$ for the controls and the two ne- phrotic groups are displayed as box plots in Fig. 3. Using either estimate of hydraulic permeability, there was little or no overlap between the ranges of $k$ obtained for the healthy controls and the ranges for either nephrotic group. The patterns obtained with $k_{\text {exp }}^{(35)}$ and $k_{\text {exp }}^{(45)}$ (not shown) were similar. These plots clearly illustrate that the hydraulic permeability was very similar in membranous and minimal change nephropathies and was strikingly lower in those groups than in healthy controls.

The fractional clearances of albumin $\left(\theta_{\mathrm{alb}}\right)$ and $\mathrm{IgG}\left(\theta_{\mathrm{IgG}}\right)$ for membranous nephropathy and minimal change nephropathy are plotted as functions of $k_{\text {model }}$ in Fig. 4 . There were significant negative correlations between either fractional clearance and $k_{\text {model }}$. The correlation coefficients between $\log \theta_{\text {alb }}$ and $k_{\text {model }}$ were $r=-0.33$ for membranous nephropathy, $r=-0.59$ for minimal change nephropathy, and $r=-0.39$ for the combined data of minimal change nephropathy and membranous nephropathy. The corresponding relationships between $\log \theta_{\mathrm{IgG}}$ and $k_{\text {model }}$ were even stronger $(r=-0.47,-0.60$, and -0.51 , respectively). Thus, the individuals with the highest fractional clearances of albumin and IgG tended to have the lowest hydraulic permeabilities. The same conclusion is reached if $\theta_{\mathrm{alb}}$ and $\theta_{\mathrm{IgG}}$ are plotted against $k_{\text {exp }}$.

\section{Discussion}

In both groups of nephrotic individuals, GFR was reduced by about $30 \%$ relative to an age-matched group of healthy controls. Among the determinants of GFR, $\pi_{\mathrm{A}}$ was depressed in the nephrotic patients while RPF was not significantly altered. These findings are in keeping with previous observations in membranous and minimal change nephropathies $(1,2,10,11)$. Given that MAP was higher in each nephrotic group than in controls, it is unlikely that glomerular capillary pressure and hence $\Delta P$ were lower in nephrotics than in controls. Thus, the only factor that can explain the reduced GFR is a decrease in the overall $K_{\mathrm{f}}$ for the two kidneys. The precise magnitude of the calculated reduction in $K_{\mathrm{f}}$ depends on what is assumed to have happened to $\Delta \mathrm{P}$. If $\Delta \mathrm{P}$ remained constant at either 35 or $40 \mathrm{mmHg}$, then $K_{\mathrm{f}}$ was reduced by roughly a factor of two to four, the greater reduction in $K_{\mathrm{f}}$ corresponding to $\Delta \mathrm{P}=35 \mathrm{mmHg}$. If $\Delta \mathrm{P}$ increased by $5 \mathrm{mmHg}$ in nephrotics, then there were approximately three- to fivefold reductions in $K_{\mathrm{f}}$. Modest increases in $\Delta \mathrm{P}$ in the nephrotic individuals are consistent with micropuncture measurements in rats with Heymann nephritis or with nephropathies induced by puromycin or adriamycin, which are regarded as experimental models for membranous nephropathy and minimal change nephropathy (23-26).

Changes in peripheral capillary surface area per glomerulus $(S)$ or in the number of functioning glomeruli are incapable of explaining the two- to fivefold reductions in $K_{\mathrm{f}}$. The percentage of globally sclerosed glomeruli measured in nephrotic individuals was similar to that observed in controls, and this sclerosis is likely due to aging. This suggests that there was little or no reduction in the number of functioning glomeruli in the membranous nephropathy and minimal change nephropathy groups. Indeed, $\geq 94 \%$ of glomerular tufts appeared nonsclerosed and widely patent by light microscopy in all groups studied. There was a tendency toward increased $S$ in both nephrotic groups, although the increase relative to controls reached statistical significance only for membranous nephropathy. Because the changes in the number of functioning glomeruli appeared to 

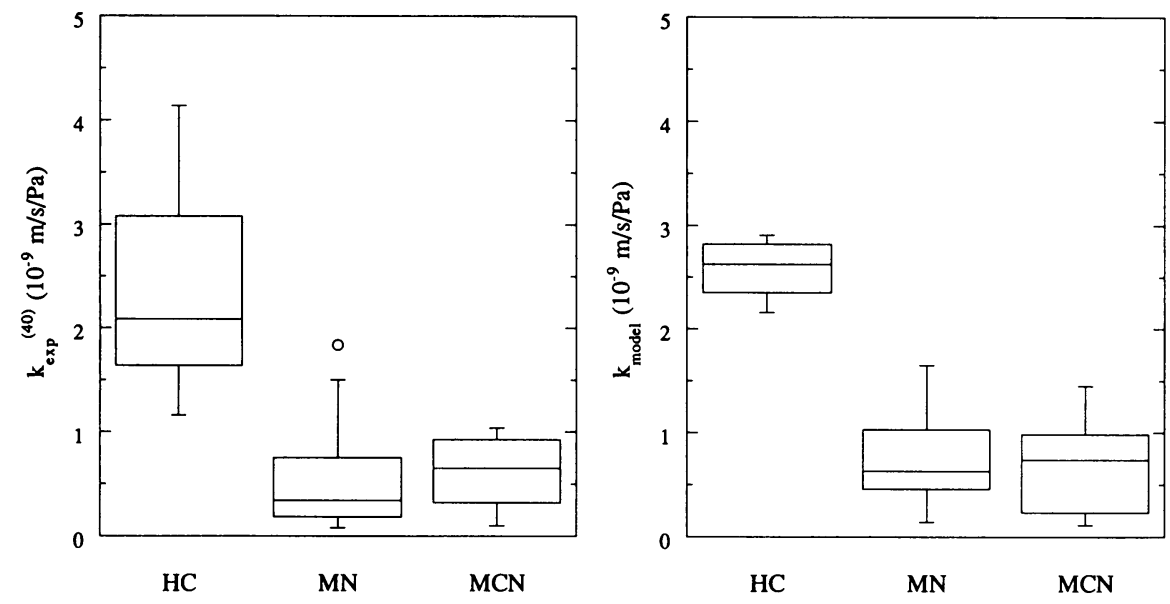

Figure 3. Quartile box plots of experimental estimates $\left(k_{\mathrm{exp}}^{(40)}\right)$ and model predictions $\left(k_{\text {model }}\right)$ of the hydraulic permeability of the glomerular capillary wall in healthy controls $(H C)$ and in membranous $(M N)$ and minimal change $(M C N)$ nephropathies. The horizontal lines comprising each box are the $25^{\text {th }}$ (lower), $50^{\text {th }}$ (middle), and $75^{\text {th }}$ (top) percentiles, respectively. In all but one case, the vertical lines represent the remainder of the range. In that case, the outlier is shown as an open symbol.

be quantitatively insignificant, and because $S$ tended to remain constant or to increase, we infer that the depression of $K_{\mathrm{f}}$ must be attributable entirely to reductions in the effective hydraulic permeability of the capillary wall $(k)$. Using GFR and its determinants to calculate the hydraulic permeability, the resulting values $\left(k_{\text {exp }}\right)$ were virtually identical in membranous nephropathy and minimal change nephropathy.

An alternative way to examine changes in hydraulic permeability is provided by the mathematical model which combines structural information and hydrodynamic calculations at the level of individual components of the glomerular capillary wall (13). This ultrastructural model does not use any information about GFR or its hemodynamic determinants, so that the resulting values of hydraulic permeability $\left(k_{\text {model }}\right)$ are entirely independent of $k_{\text {exp }}$. There are three principal assumptions of the ultrastructural model. One is that the capillary wall can be represented as having many repeating structural units as shown in Fig. 1. A second assumption is that the intrinsic hydraulic permeability (Darcy permeability) of the basement membrane is the same as that which has been measured for the isolated rat glomerular basement membrane (27). The third principal assumption is that the hydraulic resistance of the slit diaphragm can be calculated from an analysis of viscous flow through the zipperlike structure first described in rats by Rodewald and Karnovsky (21). These assumptions are supported by the fact that the predicted hydraulic permeabilities for rats are in excellent agreement with values measured by micropuncture (13).
The applicability of this model to human data is supported by the finding that $k_{\text {model }}$ accurately predicts not only the trends in $k_{\text {exp }}$ in the present study but also the absolute values in each group of subjects.

Perhaps the main value of the ultrastructural model is that it can explain findings which are not intuitively obvious. The similarity of $k_{\text {exp }}$ in minimal change nephropathy and membranous nephropathy, despite the twofold difference in basement membrane thickness $\left(\delta_{\mathrm{bm}}\right)$, is one such finding. To explain this result, we consider now the individual factors which contribute to $k$. First of all, the calculations using the ultrastructural model suggest that, in healthy controls as well as in subjects with membranous nephropathy and minimal change nephropathy, the hydraulic resistance of the endothelium is negligible, the basement membrane resistance is about $60 \%$ of the total, and the epithelial resistance accounts for the remaining $40 \%$. To examine in more detail the factors which contribute to the basement membrane resistance, a good approximation to $k_{\mathrm{bm}}$ under most conditions ${ }^{2}$ is

2. Except for one subject with membranous nephropathy and four with minimal change nephropathy, Eq. 9 yielded values of $k_{\mathrm{bm}}$ within $2 \%$ of those obtained from the more rigorous formula (Eq. 23 of Drumond and Deen [13]). For those exceptional cases, in which $\delta_{\mathrm{bm}} / W \leq 0.08$, the discrepancies were $19-37 \%$.
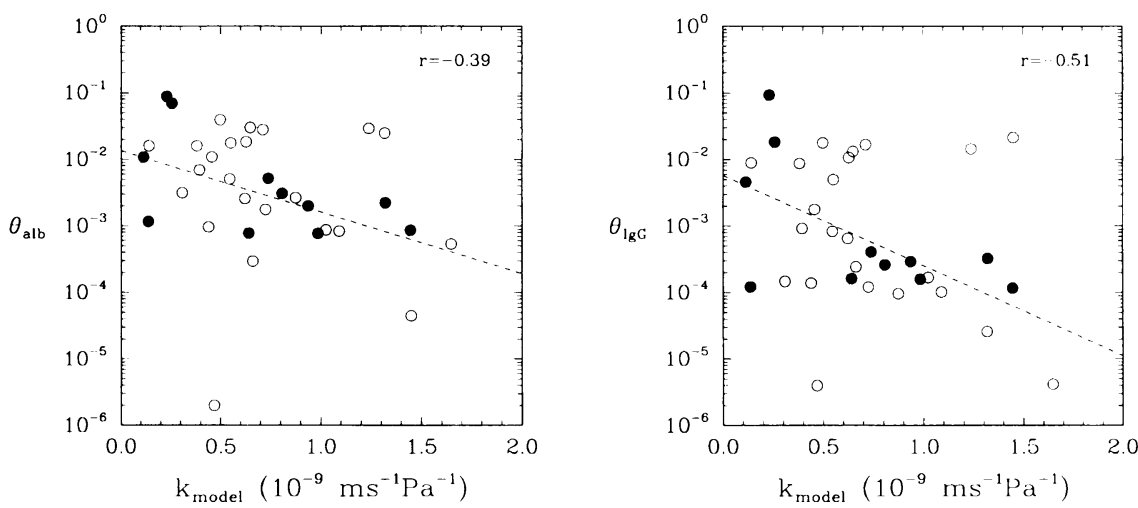

Figure 4. Individual values of the fractional clearances of albumin $\left(\theta_{\mathrm{alb}}\right.$, left $)$ and IgG $\left(\theta_{\mathrm{IgG}}\right.$, right $)$ in membranous (open symbols) and minimal change (filled symbols) nephropathies, plotted as a function of $k_{\text {model }}$. In each plot, the dashed line is the regression line for the two groups. The corresponding $r$ values are also shown. 

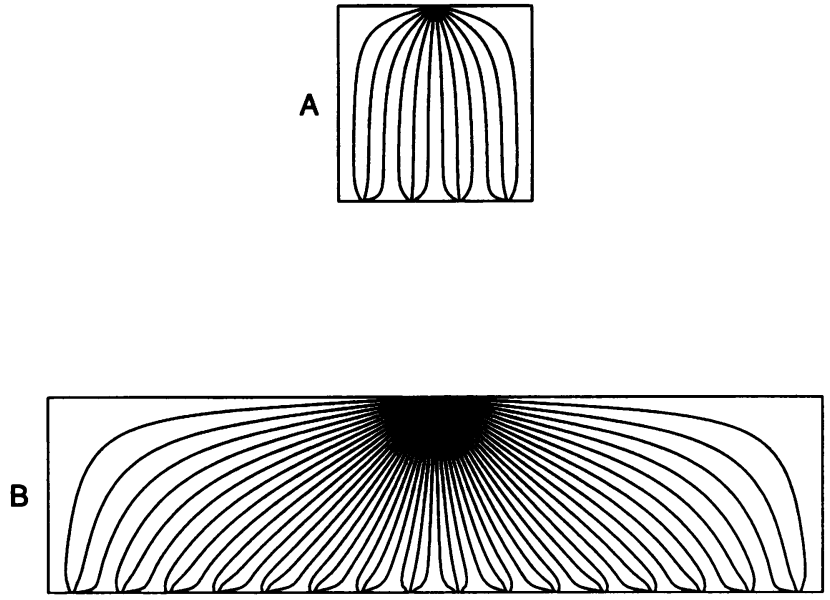

Figure 5. Streamlines in the basement membrane, illustrating the effect of changes in filtration slit frequency. $A$ is an example representative of healthy controls. In $B$ the filtration slit frequency is four times smaller than that in $A$. The basement membrane thickness, the frequency and fractional area of the fenestrae, and the width of the slit opening are the same in both panels. There are 4 fenestrae in the structural unit of $A$ and 16 in that of $B$.

$$
\begin{aligned}
k_{\mathrm{bm}} \cong \frac{K_{\mathrm{D}}}{\mu W}\left\{\frac{\delta_{\mathrm{bm}}}{W}+\frac{1}{\pi n_{\mathrm{f}}}[1.5\right. & \left.-\ln \left(2 \pi \epsilon_{\mathrm{f}}\right)\right] \\
& \left.+\frac{1}{\pi}\left[1.5-\ln \left(2 \pi \epsilon_{\mathrm{s}}\right)\right]\right\}^{-1} .
\end{aligned}
$$

The three main contributions to $k_{\mathrm{bm}}$ are the terms separated by " + " signs. The first term, involving $\delta_{\text {bm }}$, represents the resistance of "bare" basement membrane (i.e., if endothelial cells and foot processes did not cover the basement membrane surfaces). The second term, involving $n_{\mathrm{f}}$ and $\epsilon_{\mathrm{f}}$, gives the increased basement membrane resistance due to coverage by endothelial cells; and the third term, containing $\epsilon_{\mathrm{s}}$, is the increased resistance due to coverage by podocytes. It is worth noting that elementary membrane models yield only the first term in Eq. 9 and that a two- or three-dimensional representation of the flow field in the basement membrane is needed to account for the surface blockage effects (13).

In healthy controls, the first term in Eq. 9 was found to be the most important, accounting for $\sim 60 \%$ of the basement membrane resistance. That is, the basement membrane thickness is an important determinant of $k_{\mathrm{bm}}$ and $k$ in healthy individuals. By contrast, in nephrotic subjects the filtration slit frequency was reduced enough that the third term became dominant ( $\sim 60 \%$ in membranous nephropathy and $\sim 80 \%$ in minimal change nephropathy). Accordingly, $k_{\mathrm{bm}}$ in membranous nephropathy and minimal change nephropathy was determined primarily by filtration slit frequency and not by basement membrane thickness. The reason that $k_{\mathrm{bm}}$ becomes almost independent of basement membrane thickness is that, with widely separated slits, much of the flow path for filtrate within the basement membrane is parallel to the basement membrane surfaces, as is illustrated by the streamlines (i.e., lines which represent the local direction of flow) shown in Fig. 5. Fig. 5 A shows streamlines in a structural unit representative of healthy controls, and $B$ shows streamlines in a structural unit with a much smaller slit frequency, similar to that of the nephrotic subjects. For this illustration, we assumed that the basement membrane thickness is the same in both cases but that the filtration slit frequency is four times smaller in $B$ than in $A$. Consequently, the width of the structural unit in $B$ is four times that in $A$. The path length for the filtrate, which ultimately dictates the pressure drop across the basement membrane, tends to be much larger in $B$ than in $A$, even with identical basement membrane thickness. Thus, in the nephrotic groups, where the filtration slit frequency is considerably reduced relative to that in controls, the average path length and the resulting pressure drop are determined more by the slit spacing than by basement membrane thickness.

Assuming no major differences in filtration slit width or slit diaphragm structure, the hydraulic permeability of the epithelial layer $\left(k_{\text {ep }}\right)$ is governed mainly by filtration slit frequency. With both $k_{\mathrm{bm}}$ and $k_{\mathrm{ep}}$ in the nephrotic groups being determined primarily by filtration slit frequency, so is the overall value of $k$. This provides an attractive explanation for the aforementioned fact that reductions in GFR and $K_{\mathrm{f}}$ in nephrotic subjects tend to be correlated much more strongly with reductions in filtration slit frequency than with changes in basement membrane thickness (8-11).

For lack of specific data, we assumed that the total number of nephrons $(n)$ was the same in all individuals of the present study, although differences in $n$ are likely to exist. For example, it has been observed that $n$ decreases with age $(20,28)$. To minimize the effects of age differences on our results, we excluded individuals with age $\geq 60 \mathrm{yr}$. If we had excluded all subjects with ages $\geq 50 \mathrm{yr}$, the results would not have been significantly changed. Recently, it has been observed that $n$ in diabetic patients with advanced nephropathy is smaller than in healthy controls (29). If such differences exist in membranous nephropathy and minimal change nephropathy, it is possible that the correction for glomerular sclerosis used in Eq. 5 is not enough to account for the differences in $n$ between groups.

The most widely used method to determine $n$ (as well as glomerular volume, $\left.V_{G}\right)$ is that proposed by Weibel $(18,30)$. Using this method, estimates of $\boldsymbol{n}$ in healthy humans have been $\sim 2 \times 10^{6}(20)$. Recently, however, this method was criticized as being biased $(29,31)$, and a new method to determine $n$ and glomerular volume was proposed. Using this new method, a value of $n=1.2 \times 10^{6}$ was obtained (28). Since the theoretical basis of the relationships involved in the calculations of $V_{G}$ (which is related to the glomerular filtering surface area, $S$, by Eq. 3 ) and $n$ are the same, and because in this study we used the method of Weibel $(18,30)$ to determine glomerular volume, we chose to use $n=2 \times 10^{6}$ in Eq. 5 . In fact, with the method of Weibel, the product $n S$ (which appears in Eq. 5) does not involve the shape coefficient $(\beta)$ and distribution coefficient $(d)$, which are sources of bias.

The reductions in $k$ in membranous nephropathy and minimal change nephropathy appear at first to be inconsistent with the finding of considerable proteinuria, as reflected by the values of fractional albumin clearance and fractional IgG clearance. That is, one might expect that any structural changes which retard filtration of water would at least equally retard the transmural passage of proteins. One reasonable hypothesis to explain these findings is that the broadening of the foot processes (decrease of filtration slit frequency) causes changes in the structure or even entirely disrupts some of the slit diaphragms. Such changes could augment considerably the filtration of albumin and IgG, without significantly affecting the value of $k$. For example, if $5 \%$ of the slit 
diaphragms are assumed to be disrupted in membranous nephropathy and minimal change nephropathy, and if this causes the resistance of the epithelium to water flow to be negligible in $5 \%$ of the capillary wall, the mean values of $k_{\text {model }}$ would be $7.57 \times$ $10^{-10}$ and $7.22 \times 10^{-10} \mathrm{~m} / \mathrm{s} / \mathrm{Pa}$ in membranous nephropathy and minimal change nephropathy, respectively. These values are $<4 \%$ higher than those given in Table IV, not a very noticeable difference. However, given the observation that the cellular component of the capillary wall accounts for much of the overall size selectivity (32), it is entirely possible that the rupture of this small fraction of the slit diaphragms might allow the filtration of large quantities of albumin and IgG. Another possible explanation is that in membranous nephropathy and minimal change nephropathy there might have been changes in the fixed charge content of the capillary wall which, while not affecting $k$, could have greatly reduced the resistance to filtration of albumin. Charge selectivity of the glomerular barrier in healthy humans and its impairment in nephrotic subjects with either membranous or minimal change nephropathy has been demonstrated recently (33).

Another potential application of the ultrastructural model would be to use the predicted values of $k$ to calculate $K_{\mathrm{f}}$ and, together with the hemodynamic data (GFR, RPF, and $\pi_{\mathrm{A}}$ ), to estimate $\Delta \mathrm{P}$ for each individual. Because of the assumptions involved in the model, as well as in the value of nephron number used to calculate $K_{\mathrm{f}}$, any such estimates of $\Delta \mathrm{P}$ at present are highly tentative. Nonetheless, with the present data and assumptions, the mean values of the predicted $\Delta \mathrm{P}$ were $\sim 39 \mathrm{mmHg}$ in healthy controls, $\sim 36 \mathrm{mmHg}$ in membranous nephropathy, and $\sim 42$ $\mathrm{mmHg}$ in minimal change nephropathy (neglecting the value for one patient with minimal change nephropathy, which was larger than $100 \mathrm{mmHg}$ ). The value of $\Delta \mathrm{P}$ for healthy controls is quite similar to that calculated for healthy humans by an indirect method $(\Delta \mathrm{P}=36 \mathrm{mmHg})$, one based on curve fitting of the measured sieving coefficients of neutral dextran of graded size (34). Moreover, mean values for $\Delta \mathrm{P}$ predicted by $k_{\text {model }}$ for all three groups are remarkably similar to corresponding values measured directly by micropuncture in normal rats, and in rats with induced glomerular diseases which are analogues of membranous and minimal change nephropathy in humans (23-26).

To summarize, our analysis suggests that a reduction of glomerular hydraulic permeability is a major determinant of the impaired ultrafiltration capacity in membranous nephropathy and minimal change nephropathy. To elucidate the basis for the reduced hydraulic permeability, we used a recent hydrodynamic model of flow across the structural components of the glomerular capillary wall $(12,13)$. We concluded that, in both disorders, the hydraulic permeability is determined mainly by the slit spacing rather than by the basement membrane thickness. These findings point to an injury to epithelial foot processes, with an ensuing reduction in the frequency of intervening filtration slits, as the predominant cause of hypofiltration during the acute, nephrotic stage of each of these glomerular injuries.

\section{Appendix}

Because of the random angle of sectioning the glomerular capillary, the measured apparent width of a structural unit $\left(W^{\prime}\right)$ (i.e., the sum of foot process width and filtration slit width) will generally exceed the true width $(W)$. As illustrated in Fig. 6, $W^{\prime}$ depends on the angle $(\phi)$ between the filtration slits and the line of intersection of the sample plane $(\mathscr{P})$ with the outer surface of the capillary wall $(\mathscr{D})$. It can be seen that for $0 \leq \phi \leq$ $\pi, W^{\prime} \geq W$, the equality holding when $\phi=\pi / 2$.

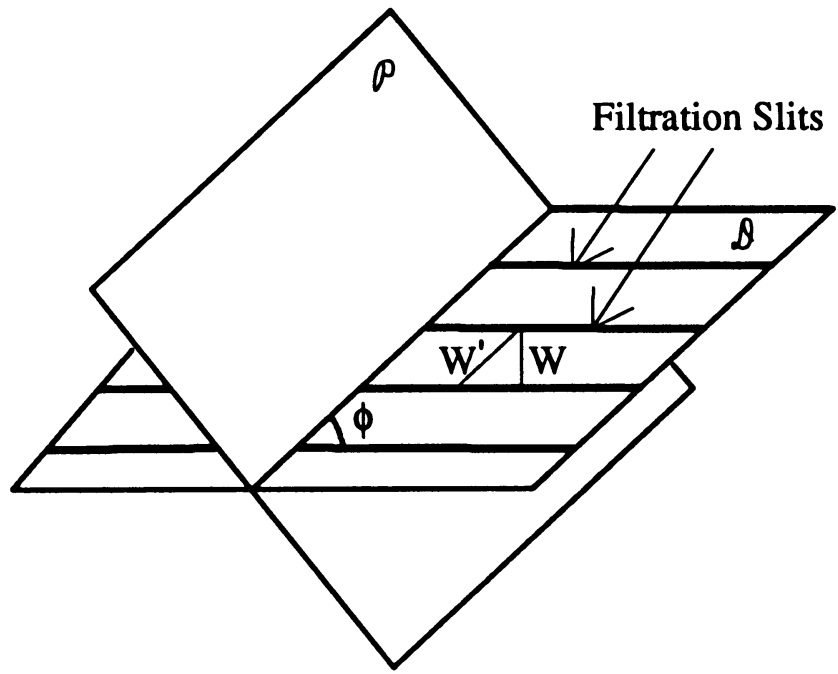

Figure 6. Schematic representation of the outer surface of the capillary wall $(\mathscr{D})$ and sample plane $(\mathscr{P})$, showing the relationship between the measured apparent width of a structural unit $\left(W^{\prime}\right)$ and the true width $(W)$.

To calculate $W$ from the measured mean width of a structural unit $\left(\bar{W}^{\prime}\right)$, we used principles of geometrical probability. In particular, our problem is closely related to that of calculating the expected length $(\sigma)$ of the chord formed by the intersection of a plane $(\mathscr{P})$ with a closed convex figure lying in another plane $(\mathscr{D})$. Using results given in Kendall and Moran (35) we obtained

$\sigma=\frac{\pi A}{P}$

where $A$ is the area of the closed convex curve, and $P$ is its perimeter. This result is equivalent to that derived by Solomon (36) for the case of a straight line intersecting a closed convex curve in a plane. Eq. Al can be applied directly to our problem by defining the closed curve as a rectangle of width $W$ and (arbitrary) length $L$, corresponding to the area occupied by one foot process and one filtration slit. Then, $\sigma=\bar{W}^{\prime}$. Neglecting edge effects (i.e., assuming $L \gg W$ ), Eq. A1 yields

$\bar{W}^{\prime}=\frac{\pi}{2} W$

which is the desired relationship between the apparent and true widths of a structural unit. This is the basis for the factor $2 / \pi$ in Eq. 6 .

Eq. A2 can be derived also as follows. As indicated by Fig. 6, the apparent width $W^{\prime}$ is related to $W$ by

$W^{\prime}=\frac{W}{\sin \phi}$.

The arithmetic mean of $W^{\prime}$ is then calculated by

$\bar{W}^{\prime}=\frac{\int_{0}^{\pi} W^{\prime} f(\phi) d \phi}{\int_{0}^{\pi} f(\phi) d \phi}$

where $f(\phi)$ is the probability density of the angle $\phi$. As shown by Solomon (36), the probability density of the angle formed by two random lines that are known to intersect within some finite region in space is given by

$f(\phi)=\frac{1}{2} \sin \phi$.

Using Eqs. A3 and A5 in Eq. A4, and performing the required integrations, one obtains again Eq. A2. 
Similarly, one can also calculate $W$ from the apparent harmonic mean width $\left(\bar{W}_{\mathrm{h}}^{\prime}\right)$ which is defined by

$\frac{1}{W_{\mathrm{h}}^{\prime}}=\frac{\int_{0}^{\pi} \frac{1}{W^{\prime}} f(\phi) d \phi}{\int_{0}^{\pi} f(\phi) d \phi}$

The result is

$\bar{W}_{\mathrm{h}}^{\prime}=\frac{4}{\pi} W$.

The derivations presented here to obtain relationships between $\bar{W}^{\prime}$ ( or $\bar{W}_{\mathrm{h}}^{\prime}$ ) and $W$ are different from that of Jensen et al. (19), who obtained the relationship we have used to calculate the "true" thickness of the basement membrane ( $\left.\delta_{\mathrm{bm}}, \mathrm{Eq} .4\right)$. To determine $\delta_{\mathrm{bm}}$ one measures the distance between two lines obtained by the intersection of the sample plane $(\mathscr{P})$ with two parallel planes $\left(\mathscr{D}^{\prime}\right.$ and $\left.\mathscr{D}\right)$ corresponding to the "inner" and "outer" surfaces of the basement membrane. The angle of interest in that case (denoted as $\theta$ ) is that between $\mathscr{P}$ and $\mathscr{D}$ (or $\mathscr{D}^{\prime}$ ), and the probability density for that angle is proportional to $\sin ^{2}$ $\theta(19)$. In contrast, $W^{\prime}$ is the distance between two points in the line of intersection of the sample plane $(\mathscr{P})$ with the plane $(\mathscr{D})$ corresponding to the outer surface of the basement membrane. The angle of interest is now the angle $(\phi)$ formed by the line of intersection of the two planes with the parallel lines in $\mathscr{D}$ corresponding to the filtration slits. This is why, contrary to the suggestion of Gundersen et al. (37), we did not use the same correction factors for $W$ and $\delta_{\mathrm{bm}}$.

\section{Acknowledgments}

This work was supported by grants from the National Institutes of Health (DK-20368 and DK-29985). M. C. Drumond is the recipient of a fellowship from Junta Nacional de Investigação Científica e Tecnológica, Programa CIENCIA, Portugal, and B. Kristal is the recipient of a fellowship from the Juvenile Diabetes Foundation International.

\section{References}

1. Guasch, A., R. K. Sibley, P. Huie, and B. D. Myers. 1992. Extent and course of glomerular injury in human membranous glomerulopathy. Am. J. Physiol. 263 (Renal Fluid Electrolyte Physiol. 32):F1034-F1043.

2. Guasch, A., H. Hashimoto, R. K. Sibley, W. M. Deen, and B. D. Myers. 1991. Glomerular dysfunction in nephrotic humans with minimal changes or focal glomerulosclerosis. Am. J. Physiol. 260 (Renal Fluid Electrolyte Physiol. 29):F728-F737.

3. Myers, B. D., A. Chagnac, H. Golbetz, L. Newton, S. Strober, and R. K. Sibley. 1991. Extent of glomerular injury in active and resolving lupus nephritis: a theoretical analysis. Am. J. Physiol. 260 (Renal Fluid Electrolyte Physiol. 29):F717-F727.

4. Austin, S. M., J. S. Lieberman, L. D. Newton, M. Mejia, W. A. Peters, and B. D. Myers. 1993. Slope of serial GFR and the progression of diabetic glomerular disease. J. Am. Soc. Nephrol. 3:1358-1370.

5. Scandling, J. D., V. M. Black, W. M. Deen, and B. D. Myers. 1992. Glomerular permselectivity in healthy and nephrotic humans. Necker's Seminar in Nephrology. Adv. Nephrol. 21:159-176.

6. Mauer, S. M., M. W. Steffes, E. N. Ellis, D. E. R. Sutherland, D. M. Brown, and F. C. Goetz. 1984. Structural-functional relationships in diabetic nephropathy. J. Clin. Invest. 74:1143-1155.

7. Osterby, R., H. J. G. Gundersen, A. Horlyck, J. P. Koustrup, G. Nyberg, and G. Westberg. 1983. Diabetic glomerulopathy: structural characteristics of the early and advanced stages. Diabetes. 32(Suppl. 2):79-82

8. Bohman, S. O., G. Jaremko, A. B. Bohlin, and U. Berg. 1984. Foot proces fusion and glomerular filtration rate in minimal change nephrotic syndrome. Kidney Int. 25:696-700.

9. Ellis, E. N., M. W. Steffes, B. Chavers, and S. M. Mauer. 1987. Observations of glomerular epithelial cell structure in patients with type I diabetes mellitus. Kidney Int. 32:736-741.

10. Guasch, A., and B. D. Myers. 1994. Determinants of glomerular hypofiltration in nephrotic patients with minimal change nephropathy. J. Am. Soc Nephrol. 4:1571-1581.
11. Shemesh, O., J. C. Ross, W. M. Deen, G. W. Grant, and B. D. Myers 1986. Nature of the glomerular capillary injury in human membranous glomerulopathy. J. Clin. Invest. 77:868-877.

12. Drumond, M. C., and W. M. Deen. 1994. Stokes flow through a row of cylinders between parallel walls: model for the glomerular slit diaphragm. $J$. Biomech. Eng. 116:184-189.

13. Drumond, M. C., and W. M. Deen. 1994. Structural determinants of glomerular hydraulic permeability. Am. J. Physiol. 266 (Renal Fluid Electrolyte Physiol. 35):F1-F12.

14. Battilana, C., H. Zhang, R. Olshen, L. Wexler, and B. D. Myers. 1991 PAH extraction and the estimation of plasma flow in the diseased human kidney. Am. J. Physiol. 261 (Renal Fluid Electrolyte Physiol. 30):F726-F733.

15. Cannan-Kuhl, S., E. S. Venkatraman, S. I. B. Ernst, R. A. Olshen, and B. D. Myers. 1993. Relationships among protein and albumin concentrations and oncotic pressure in nephrotic plasma. Am. J. Physiol. 264 (Renal Fluid Electrolyte Physiol. 33):F1052-F1059.

16. Deen, W. M., C. R. Robertson, and B. M. Brenner. 1972. A model of glomerular ultrafiltration in the rat. Am. J. Physiol. 223:1178-1183.

17. Maddox, D. A., W. M. Deen, and B. M. Brenner. 1991. Glomerular Filtration. In Handbook of Physiology: Renal Physiology. E. E. Windhager, editor. American Physiological Society, Oxford University Press, New York. 545-638.

18. Weibel, E. R. 1979. Stereological Methods. Vol. 1: Practical Methods for Biological Morphometry. Academic Press, London.

19. Jensen, E. B., H. J. G. Gundersen, and R. Osterby. 1979. Determination of membrane thickness distribution from orthogonal intercepts. J. Microsc. (Oxf.) 115:19-33.

20. Dunnill, M. S., and W. Halley. 1973. Some observations on the quantitative anatomy of the kidney. J. Pathol. 110:113-121.

21. Rodewald, R., and M. J. Karnovsky. 1974. Porous substructure of the glomerular slit diaphragm in the rat and mouse. J. Cell Biol. 60:423-433.

22. Lane, P. H., M. W. Steffes, and S. M. Maurer. 1992. Estimation of glomerular volume: a comparison of four methods. Kidney Int. 41:1085-1089.

23. Ichikawa, I., J. R. Hoyer, W. M. Seiler, and B. M. Brenner. 1982. Mechanism of glomerulotubular balance in the setting of heterogeneous glomerular injury. J. Clin. Invest. 69:185-198.

24. Yoshioka, T., H. G. Rennke, D. J. Salant, W. M. Deen, and I. Ichikawa 1987. Role of abnormally high transmural pressure in the permselectivity defect of glomerular capillary wall: a study in early passive Heymann nephritis. Circ. Res. 61:531-538.

25. Scholey, J. W., P. L. Miller, H. G. Rennke, and T. W. Meyer. 1989. Effect of converting enzyme inhibition on the course of adriamycin-induced nephropathy Kidney Int. 36:816-822.

26. Anderson, S., J. R. Diamond, M. J. Karnovsky, and B. M. Brenner. 1988 Mechanisms underlying transition from acute glomerular injury to late glomerular sclerosis in a rat model of nephrotic syndrome. J. Clin. Invest. 82:1757-1768.

27. Daniels, B. S., E. B. Hauser, W. M. Deen, and T. H. Hostetter. 1992. Glomerular basement membrane: in vitro studies of water and protein permeability. Am. J. Physiol. 262 (Renal Fluid Electrolyte Physiol. 31):F919-F926.

28. Nyengaard, J. R., and T. F. Bendtsen. 1992. Glomerular number and size in relation to age, kidney weight, and body surface in normal man. Anat. Rec. 232:194-201.

29. Bendtsen, T. F., and J. R. Nyengaard. 1992. The number of glomeruli in type 1 (insulin-dependent) and type 2 (non-insulin-dependent) diabetic patients. Diabetologia. 35:844-850.

30. Weibel, E. R., and D. M. Gomez. 1962. A principle for counting tissue structures on random sections. J. Appl. Physiol. 17:343-348.

31. Bendtsen, T. F., and J. R. Nyengaard. 1989. Unbiased estimation of particle number using sections: an historical perspective with special reference to the stereology of glomeruli. J. Microsc. 153:93-102.

32. Daniels, B. S., W. M. Deen, G. Mayer, T. Meyer, and T. H. Hostetter. 1993. Glomerular permeability barrier in the rat. Functional assessment by in vitro methods. J. Clin. Invest. 92:929-936.

33. Guasch, A., W. M. Deen, and B. D. Myers. 1993. Charge selectivity of the glomerular filtration barrier in healthy and nephrotic humans. J. Clin. Invest. 92:2274-2282.

34. Myers, B. D., C. Peterson, C. R. Molina, S. J. Tomlanovich, L. D. Newton, R. Nitkin, H. Snadler, and F. Murad. 1988. Role of cardiac atria in the human renal response to changing plasma volume. Am. J. Physiol. 254 (Renal Fluid Electrolyte Physiol. 23):F562-F573.

35. Kendall, M. G., and P. A. P. Moran. 1963. Geometrical Probability. Charles Griffin \& Co., London. 79-80.

36. Solomon, H. 1978. Geometric Probability. Society for Industrial and Applied Mathematics, Philadelphia. 174 pp.

37. Gundersen, H. J. G., T. Seefeldt, and R. Osterby. 1980. Glomerular epithelial foot processes in normal man and rats. Cell Tissue Res. 205:147-155. 